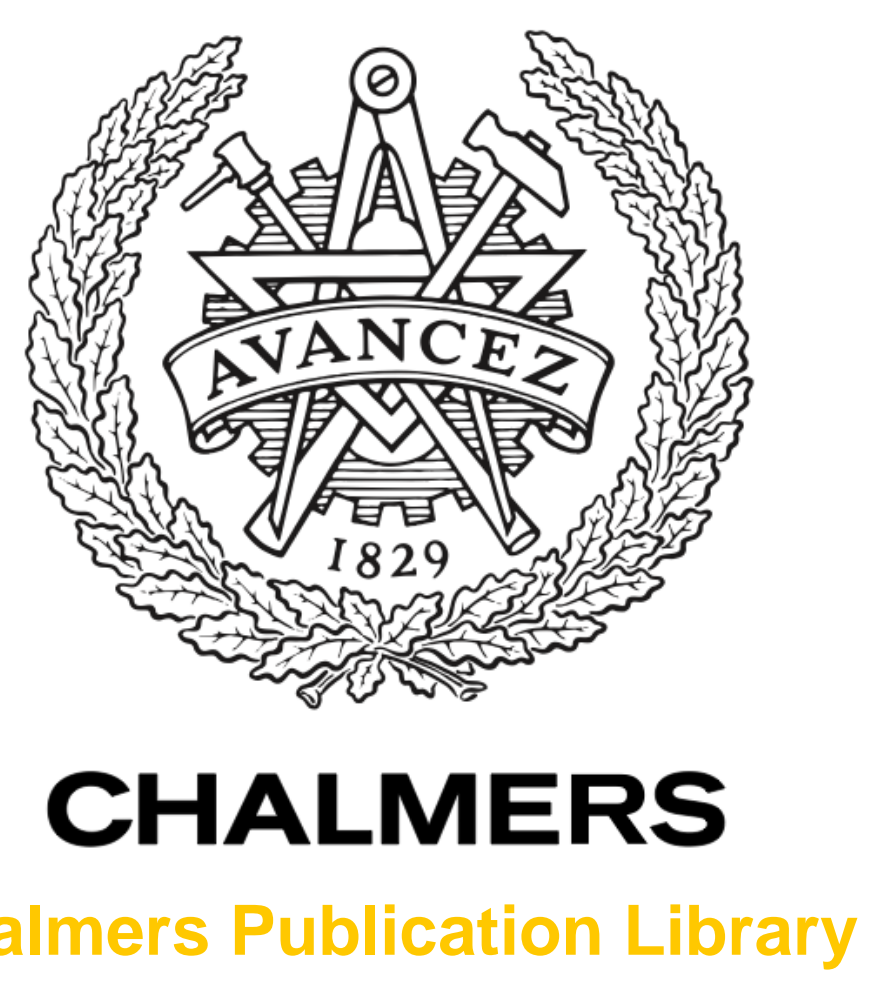

Chalmers Publication Library

\author{
Modelling the interior sound field of a railway vehicle using statistical energy \\ analysis
}

This document has been downloaded from Chalmers Publication Library (CPL). It is the author's version of a work that was accepted for publication in:

Applied Acoustics (ISSN: 0003-682X)

Citation for the published paper:

Forssén, J. ; Tober, S. ; Can Corakci, A. (2012) "Modelling the interior sound field of a railway vehicle using statistical energy analysis". Applied Acoustics, vol. 73(4), pp. 307311.

http://dx.doi.org/10.1016/j.apacoust.2011.09.012

Downloaded from: http://publications.lib.chalmers.se/publication/156087

Notice: Changes introduced as a result of publishing processes such as copy-editing and formatting may not be reflected in this document. For a definitive version of this work, please refer to the published source. Please note that access to the published version might require a subscription.

Chalmers Publication Library (CPL) offers the possibility of retrieving research publications produced at Chalmers University of Technology. It covers all types of publications: articles, dissertations, licentiate theses, masters theses, conference papers, reports etc. Since 2006 it is the official tool for Chalmers official publication statistics. To ensure that Chalmers research results are disseminated as widely as possible, an Open Access Policy has been adopted.

The CPL service is administrated and maintained by Chalmers Library. 


\section{Modelling the interior sound field of a railway vehicle using statistical energy analysis}

Jens Forssénn ${ }^{\mathrm{a}}$, Stefan Tober ${ }^{\mathrm{a}, 1}$, Ata Can Corakcia ${ }^{\text {, Anders Frid }}{ }^{\mathrm{b}}$, Wolfgang Kropp ${ }^{\mathrm{a}}$

aApplied Acoustics, Chalmers University of Technology, SE 41296 Göteborg, Sweden.

Email: jens.forssen@chalmers.se, corakci@alumni.chalmers.se,

wolfgang.kropp@chalmers.se

bBombardier Transportation, PASSENGERS Division, Acoustics \& Vibration, Östra Ringvägen 2, 72173 Västerås, Sweden.

Email: anders.r.frid@se.transport.bombardier.com

1Present address: MBM Industry \& Rail Tech GmbH, A-3002 Purkersdorf, Austria. Email: stefan.tober@mbm-industrietechnik.at

Corresponding author: Jens Forssén, Applied Acoustics, Chalmers University of

Technology, SE 41296 Göteborg, Sweden. Tel: 4631772 2200. Fax: 46317722212.

Email: jens.forssen@chalmers.se

\section{Abstract}

The sound field in train compartments, treated as a series of connected air cavities, is modelled using statistical energy analysis, SEA. For the case under study, with five cavities in series and the source in the second cavity, a closed-form solution is obtained. An adjusted SEA model is used to predict the rate of spatial decay within a cavity. The SEA model is validated using results from a ray tracing method and from scale model measurements. For the octave bands $500-4000 \mathrm{~Hz}$, good agreement is shown between the results from measurements, the ray tracing and the SEA model, for the two saloons closest to the source cavity (a vestibule). The SEA model was shown to slightly underestimate the rate of spatial decay within a cavity. It is concluded that a reasonable cause is the additional diffusion due to the seating.

Keywords: Statistical Energy Analysis, Air Cavity, Train Compartment, Spatial Decay, Ray Tracing, Scale Model.

\section{Introduction}

The sound field in train compartments has become an important part of the design process over the past years. Knowledge about the sound perceived by the passenger is not only interesting in terms of avoiding annoyance from high noise levels, it also helps to build a quality image of the product. Therefore it is desirable for the train industry to predict the interior sound field as early as possible in the design process. At an early stage, sound design measures can be relatively simple and cheap whereas changes to an already built train are mostly very hard to implement and can be very cost-intensive.

This research was initiated to investigate the sound distribution in train interiors, in order to provide a solid basis for the implementation of the acquired knowledge in future prediction tools. The part of the work that is the main focus of the current paper deals with the sound field modelling using statistical energy analysis, SEA. For typical cases of rail vehicle interiors, the problem formulation consists of air cavities in series. In the present case, five air cavities, consisting of three saloons and two vestibules, are 
connected by openings. The source is located in one of the vestibules, at a relatively low height and near to the outer wall, in order to simulate the traction and rolling noise transmitted to the interior through the entrance doors. At cruising speed this transmitted noise has a broadband character typically spanning a wide frequency range of 500-2000 Hz. The elongated shape of the saloons together with the sound absorptive surface materials lead to a sound decay along the length of the saloon. This uneven distribution of energy within a room constitutes a departure from the usual assumptions of diffuse field underlying SEA modelling. Therefore an adjustment of the usual SEA modelling has been applied. To validate the SEA modelling, results from a scale model measurement as well as from a ray-tracing method are used. A more detailed description of the scale model measurements than what is presented below is available in [1].

\section{Method}

\subsection{Scale model measurements}

A scale model was manufactured of a complete car of a Regina train, a regional train built by Bombardier Transportation. Using a model scale of 1:5 and a maximum frequency of the measurement system of $20 \mathrm{kHz}$, the corresponding maximum frequency in full scale is $4000 \mathrm{~Hz}$. (The sound frequency and the geometrical dimensions are in the following presented in full scale unless otherwise explicitly stated.) The analysis is made for the third-octave bands $125-4000 \mathrm{~Hz}$. The measurement system consisted of an Agilent VXI station (VXI E8408A Mainframe) analyzing the signals from a set of calibrated microphones and a noise source using an in-house developed Matlab script at Applied Acoustics, Chalmers. Electret condenser microphones from Panasonic (Type WM60) were used, with an outer diameter of $6 \mathrm{~mm}$. A cubical sound source was specially manufactured using six $20 \mathrm{~mm}$ dome tweeters. The resulting sound source had an edge length of $55 \mathrm{~mm}$ and showed good directivity characteristics. The excitation signal was random noise produced by the VXI station, amplified by a NAD 310 series. The output noise signal was looped back to the analyzer as reference.

The scale model was built from medium-density fiberboard (MDF) and acrylic glass (Makrolon®). The final scale model is $4.6 \mathrm{~m}$ long and shown in Figs. 1 and 2. The five cavities, from left to right in the drawing, are: Saloon 1, Vestibule 1, Saloon 2, Vestibule 2 and Saloon 3. The corresponding lengths are 5.8, 1.7, 7.6, 1.7 and $5.8 \mathrm{~m}$. The height is $2.2 \mathrm{~m}$ and the width is $2.9 \mathrm{~m}$ for all five cavities. Between each cavity the opening is $0.75 \mathrm{~m}$ wide and $2.2 \mathrm{~m}$ high. 

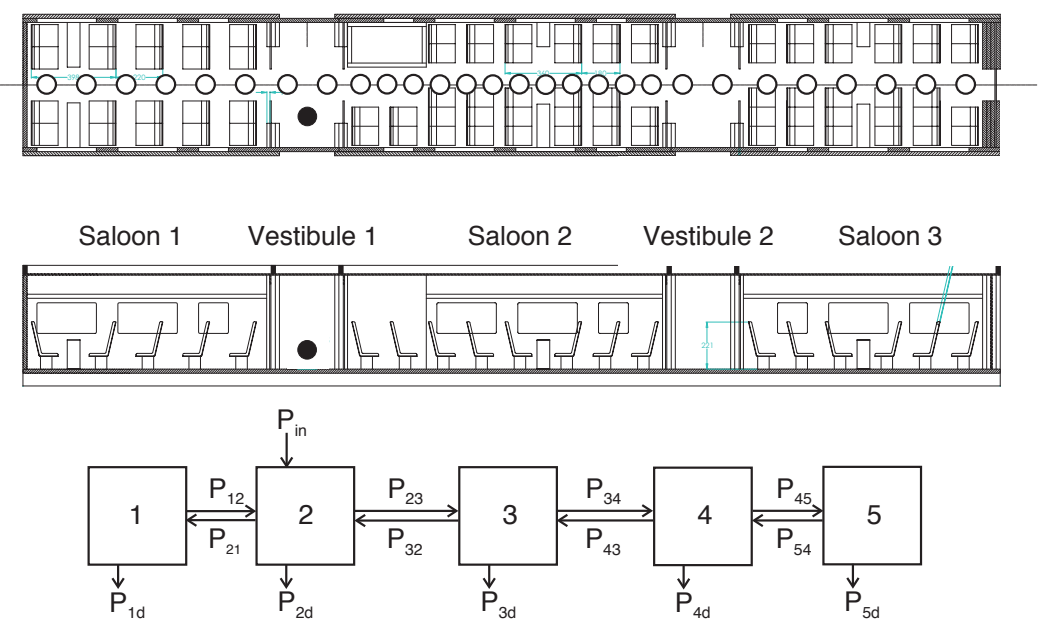

Figure 1. Top: Drawing of scale model. Below: block diagram of the SEA model with $P_{\text {in }}$ the input power to subsystem 2 (Vestibule 1), $P_{i, j}$ the power transfer between subsystems and $P_{i d}$ the losses.

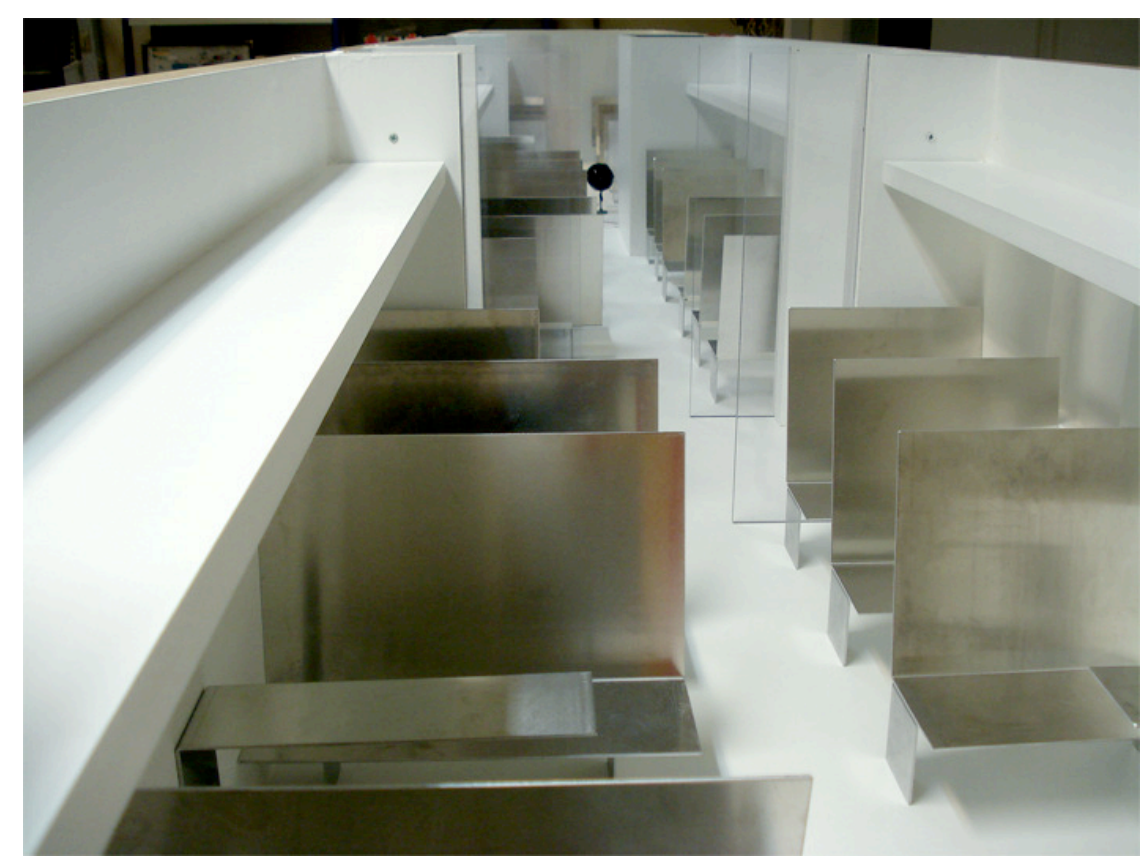

Figure 2. Photo of the interior of the scale model.

The scale model was used for both measuring the sound decay throughout the train compartments and for measuring reverberation times. The aim of the reverberation time measurements was twofold: first, by trial-and-error, finding and applying absorber materials on the surfaces to reach a good agreement with available reverberation time data for a real Regina train compartment; and second, estimating the different absorption coefficients of the applied absorber materials, which is needed for the raytracing modelling. 
The reverberation time measurements were made in Saloon 1, with the opening to Vestibule 1 sealed off with an MDF board, thereby creating a small reverberation chamber. Analogous to the standardized setup for sound absorption measurement in a reverberation room (ISO 354-1985), different placements of loudspeaker and microphones were used. With an array of five microphones, 45 different combinations of source and microphone positions were measured and averaged to give one reverberation time curve. To get the reverberation time, the $-10 \mathrm{~dB}$ drop in energy decay was found and extrapolated to get the standardized reverberation time, $T_{60}$. For that, a Matlab script was made, using Schroeder's backward integration of the impulse response (see e.g. [2]), where the impulse responses were estimated from the measured frequency responses. (It can be noted that the decay curves did not show double slopes, but clear single slope behaviour.) The final best fit to the real Regina data was obtained with $100 \mathrm{~mm}$ thick mineral wool in the ceiling with a surface area of $5.7 \times 1.95 \mathrm{~m}^{2}$, covered by a thin foil to reduce the high-frequency absorption (effective from about $1 \mathrm{kHz}$ ), in combination with a $75 \mathrm{~mm}$ thick foam on the seats. The results are displayed in Fig. 3 as average absorption coefficient, $\alpha_{\mathrm{av}}$, calculated using the Sabine formula: $A=0.16 V / T_{60}$, where $A=S_{\text {room }} \alpha_{\text {av }}$, with $S_{\text {room }}$ the total surface area of the room, and where $V$ is the room volume. The two upper curves in Fig. 3 are for the chosen absorption in the scale model and for the absorption calculated from reverberation data obtained for a real Regina train (see figure legend). The lowest curve shows the results without any absorption materials attached, which is later used in the modelling of the vestibules.

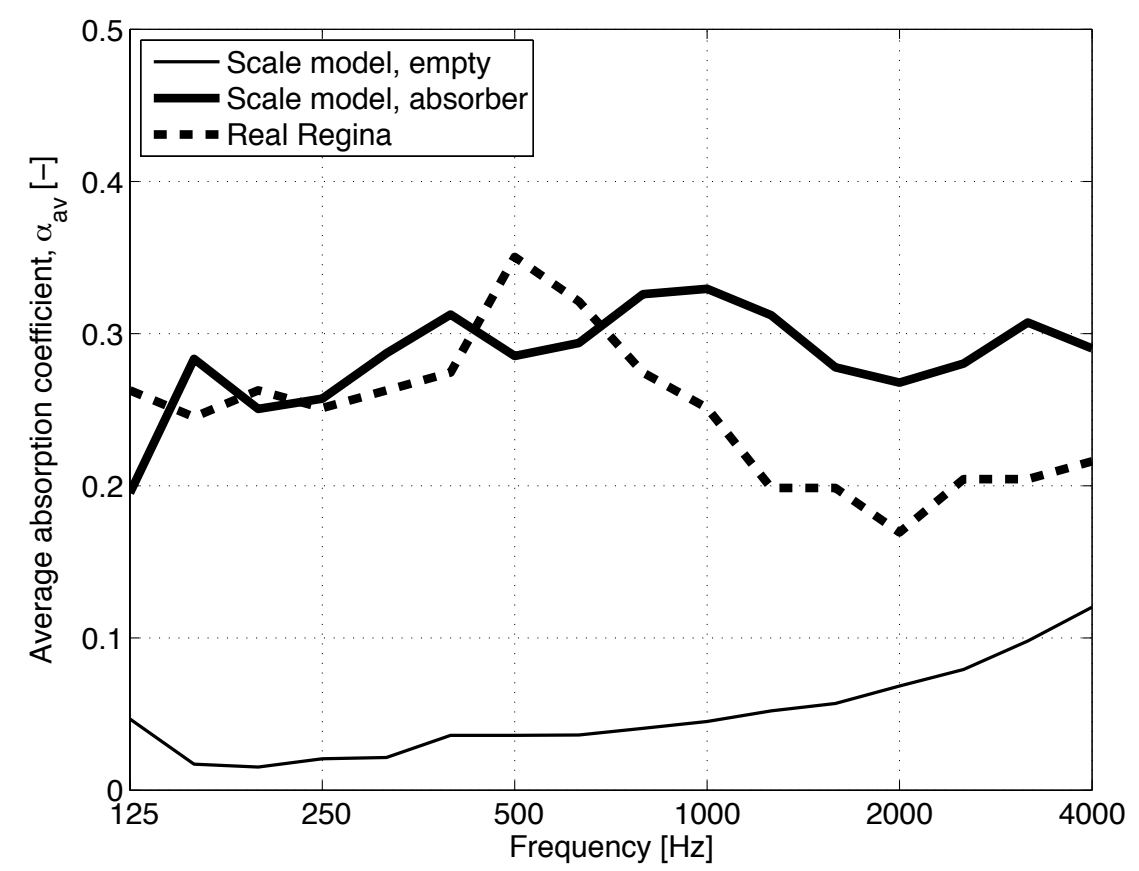

Figure 3. Measured average absorption coefficients as function of frequency.

When measuring the sound field throughout the five cavities, microphones were positioned along a single line at a height of $1.2 \mathrm{~m}$ and at a distance of $1.2 \mathrm{~m}$ from the outer wall. The source was located in Vestibule 1, at height $0.3 \mathrm{~m}$, at a distance of $0.75 \mathrm{~m}$ from the wall of Saloon 2, and at a distance of $0.6 \mathrm{~m}$ from the outer wall, on the same side as the microphone line. The spatial resolution of the measurement positions was about $1 \mathrm{~m}$ for all cavities except for Saloon 2 where $0.5 \mathrm{~m}$ was used. This resulted in six 
points for Saloons 1 and 3, twelve points for Saloon 2, and two points in each vestibule. Results were measured in the third-octave bands $125-4000 \mathrm{~Hz}$, from which the octave band results were obtained, i.e. at 125, 250, 500 1000, 2000 and 4000 Hz. However, due to a fault in the planning of the measurement setup, the complete frequency range was not fully covered; the results for the octave band $4 \mathrm{kHz}$ could only be based on the result from the single third-octave band $4 \mathrm{kHz}$ and the results for the octave band $125 \mathrm{~Hz}$ could only be based on the results from the two third-octave bands 125 and $160 \mathrm{~Hz}$.

\subsection{Ray-tracing method}

For the ray-tracing modelling the software Odeon ${ }^{\circledR}$ version 9.1 was used. The full-scale geometry was imported to Odeon, after drawings being created in commercial 3-D software. The interior detail geometries were adjusted to give a resulting sound field that approximated the sound field of the scale model measurements as well as possible. A point source was defined at the same position as where the source was placed during the scale model measurements. To achieve acceptable accuracy, two thirds of the longest reverberation time was used as impulse response length together with 33204 rays. The resulting sound field and reverberation times were calculated for the octave bands $125-4000 \mathrm{~Hz}$. Concerning numerical cost, in addition to the efforts needed to set up the ray-tracing model, the calculation time was about 30 minutes.

\subsection{Statistical energy analysis, SEA}

The three saloons and the two vestibules constitute the five subsystems of the SEA model (see Figure 1.). (For the fundamentals of SEA modelling the reader is referred to e.g. [3].) For the SEA model to be valid, the number of modes should be large enough in a frequency band of interest at the same time as the modal density in relation to the damping not being too low, i.e. a sufficiently large modal overlap factor. For sound fields in rooms, the latter requirement is analogous to being above the Schroeder frequency, $f_{S}=2000 \sqrt{T_{60} / V}$, where $V$ is the volume of the room. For the saloons, the Schroeder frequency is about $200 \mathrm{~Hz}$, whereas for the vestibules, the Schroeder frequency is increased due to the smaller volume and lower absorption, to about $1 \mathrm{kHz}$. Concerning the number of modes in a frequency band of interest, a limiting condition of ten modes per third-octave is satisfied above about $150 \mathrm{~Hz}$ for the saloons with a further increase to about $250 \mathrm{~Hz}$ for the vestibules, due to the smaller volume. (Concerning the expected first eigenfrequencies, they are at about 30 and $60 \mathrm{~Hz}$ for the saloon and the vestibule, respectively.)

With $E_{i}, i=1 \ldots 5$, the energy of the respective subsystem and $P_{\text {in }}$ the power input to the second subsystem, the power balance can be formulated as

$$
\left[\begin{array}{ccccc}
-\eta_{1} & \eta_{2,1} & 0 & 0 & 0 \\
\eta_{1,2} & -\eta_{2} & \eta_{3,2} & 0 & 0 \\
0 & \eta_{2,3} & -\eta_{3} & \eta_{4,3} & 0 \\
0 & 0 & \eta_{3,4} & -\eta_{4} & \eta_{4,5} \\
0 & 0 & 0 & \eta_{5,4} & -\eta_{5}
\end{array}\right]\left[\begin{array}{c}
E_{1} \\
E_{2} \\
E_{3} \\
E_{4} \\
E_{5}
\end{array}\right]=\left[\begin{array}{c}
0 \\
-P_{i n} / 2 \pi f \\
0 \\
0 \\
0
\end{array}\right],
$$

where $f$ is the frequency, $\eta_{i}$ is the loss factor of subsystem $i$ (fulfilling $P_{i d}=2 \pi f E_{i} \eta_{i}$, where $P_{i d}$ is the dissipated power from subsystem $i$ ) and $\eta_{i, j}$ is the coupling loss factor 
between subsystems $i$ and $j$ (fulfilling $P_{i, j}=2 \pi f E_{i} \eta_{i, j}$, where $P_{i, j}$ is the power flow from subsystem $i$ to subsystem $j$ ). The loss factors are related to the average absorption coefficients, $\alpha_{\mathrm{av}, i}$, according to

$$
\eta_{i}=\frac{c S_{\mathrm{room}, i} \alpha_{\mathrm{av}, i}}{8 \pi f V_{i}}
$$

where $c$ is the sound speed. The coupling loss factors can be written as

$$
\eta_{i, j}=\frac{c S_{i, j}}{8 \pi f V_{i}}
$$

where $S_{i, j}$ is the size of the opening between cavities $i$ and $j$.

To calculate the acoustic energy relative to the source cavity, the equations could be divided by $E_{2}$. Then the equation corresponding to the source cavity does not need to be included, i.e. the second row of Eq. (1). (The absolute energy of the source cavity can be calculated at a later stage if it is of interest.) The resulting equation system can be written as

$$
\left[\begin{array}{cccc}
\eta_{1} & 0 & 0 & 0 \\
0 & \eta_{3} & -\eta_{4,3} & 0 \\
0 & -\eta_{3,4} & \eta_{4} & -\eta_{4,5} \\
0 & 0 & -\eta_{5,4} & \eta_{5}
\end{array}\right]\left[\begin{array}{c}
E_{1} / E_{2} \\
E_{3} / E_{2} \\
E_{4} / E_{2} \\
E_{5} / E_{2}
\end{array}\right]=\left[\begin{array}{c}
\eta_{2,1} \\
\eta_{2,3} \\
0 \\
0
\end{array}\right]
$$

In the reduced equation system (Eq. 4), the first subsystem is connected only to the second subsystem and the corresponding equation (i.e. the first row of Eq. 4) can be solved separately, giving $E_{1} / E_{2}=\eta_{2,1} / \eta_{1}$. The remaining part of the equation system can be solved analytically, resulting in:

$$
\left[\begin{array}{c}
E_{3} / E_{2} \\
E_{4} / E_{2} \\
E_{5} / E_{2}
\end{array}\right]=\frac{\eta_{2,3}}{\eta_{3}\left(\eta_{4} \eta_{5}-\eta_{5,4} \eta_{4,5}\right)-\eta_{3,4} \eta_{4,3} \eta_{5}}\left\lfloor\begin{array}{c}
\eta_{5} \eta_{4}-\eta_{5,4} \eta_{4,5} \\
\eta_{5} \eta_{3,4} \\
\eta_{3,4} \eta_{4,5}
\end{array}\right] .
$$

The sound pressure level relative to the source cavity, $L_{p, i, \text { re } 2}$, is found after normalizing with the volume:

$$
L_{p, i, \mathrm{re} 2}=10 \log \left(\frac{E_{i} V_{2}}{E_{2} V_{i}}\right) .
$$

The usual SEA solution, as presented above, describes the average energy of each subsystem. To describe a spatially varying energy density within a subsystem, e.g. the decay along the corridor shaped saloons of the current study, some kind of correction is needed. In the work behind this paper, different approaches were tested. The note by Fahy [4] discusses why it is possible to subdivide a single air cavity into multiple SEA subsystems for some cases. However, the main emphasis there is on the compartment of 
light road vehicles, and it is stated that application to extended volumes such as train and aircraft compartments may be more problematic. It appears that, when using an unaltered formulation of the coupling loss factors, a further subdivision of an elongated room into smaller subsystems will lead to an overestimation of the rate of spatial decay due to an underestimation of the input to the neighbouring subsystem. Therefore this approach is not used here. Another approach, also using a subdivision of the cavity, forces the values of the coupling loss factors to attain an a priori known spatial decay [5]. This approach seems unnecessary, at least for the present problem, since, if the spatial decay throughout the cavity is known, there is no need to subdivide the cavity. Instead, we use here the result from the usual SEA modelling (as in Eqs. 1-6) but within each cavity, the spatial decay is adjusted using formulas known from literature on sound decay in corridors. The spatial decay is normalized so that the total energy of each cavity is unchanged. The decay used here can be written in terms of $\mathrm{dB}$ per meter, as from Ref. [6]:

$$
\Delta L_{p}=\frac{10}{\ln 10} \frac{\pi}{8} \frac{U \alpha}{S}
$$

Here $U$ is the perimeter length and $S$ is the cross-sectional area, i.e. here $U=2(2.2+2.9) \mathrm{m}$ and $S=2.2 \cdot 2.9 \mathrm{~m}^{2}$. An alternative formula is obtained when $\pi / 8$ in Eq. (7) is exchanged for $1 / \pi$ [7]; the difference for the present case being negligible. The above formula (Eq. 7 ) is used for the three saloons, whereas the sound fields are assumed to be spatially invariant within each vestibule, since they do not have an elongated shape and furthermore have lower absorption. In addition, to model the effect of the spatial decay in Saloon 2 on the input power to Vestibule 2 and Saloon 3, the levels there have been lowered by the difference between the spatially decayed level at the end of Saloon 2 and the average level.

\section{Results}

The sound pressure level (SPL) is plotted as function of length along the train car relative to the sound pressure level of Vestibule 1 where the source is located (i.e. subsystem 2 of the SEA analysis). For the SPL, Eq. (6) is used, with a correction of the spatial decay of the saloons according to Eq. (7). The results of the SEA modelling are shown in Fig. 4 together with those of the ray-tracing method and the scale model measurements. The spatial resolution of the SEA and ray tracing results were chosen to match that of the scale model measurements. For the saloons, also the spatial energy average is shown. In the six plots, for the different octave bands, it can be seen that the general agreement between measurements and modelling is good except for the lowest frequencies of $125 \mathrm{~Hz}$ and $250 \mathrm{~Hz}$. From $500 \mathrm{~Hz}$ and above, the ray-tracing results follow well the spatial decay shown by the measured results throughout Saloons 1 and 2, whereas the results predicted by the SEA model slightly underestimates the rate of decay, while still giving a good prediction of the average energy. For Vestibule 2, which starts at a length of about $15 \mathrm{~m}$, a fairly good agreement is shown, from $500 \mathrm{~Hz}$ and above, between the measurements and the modelling using both SEA and ray-tracing. For Saloon 3, the modelling shows larger deviations, however probably of lesser importance in practice since these relatively low levels are likely to be dominated by other sources at closer distance. 

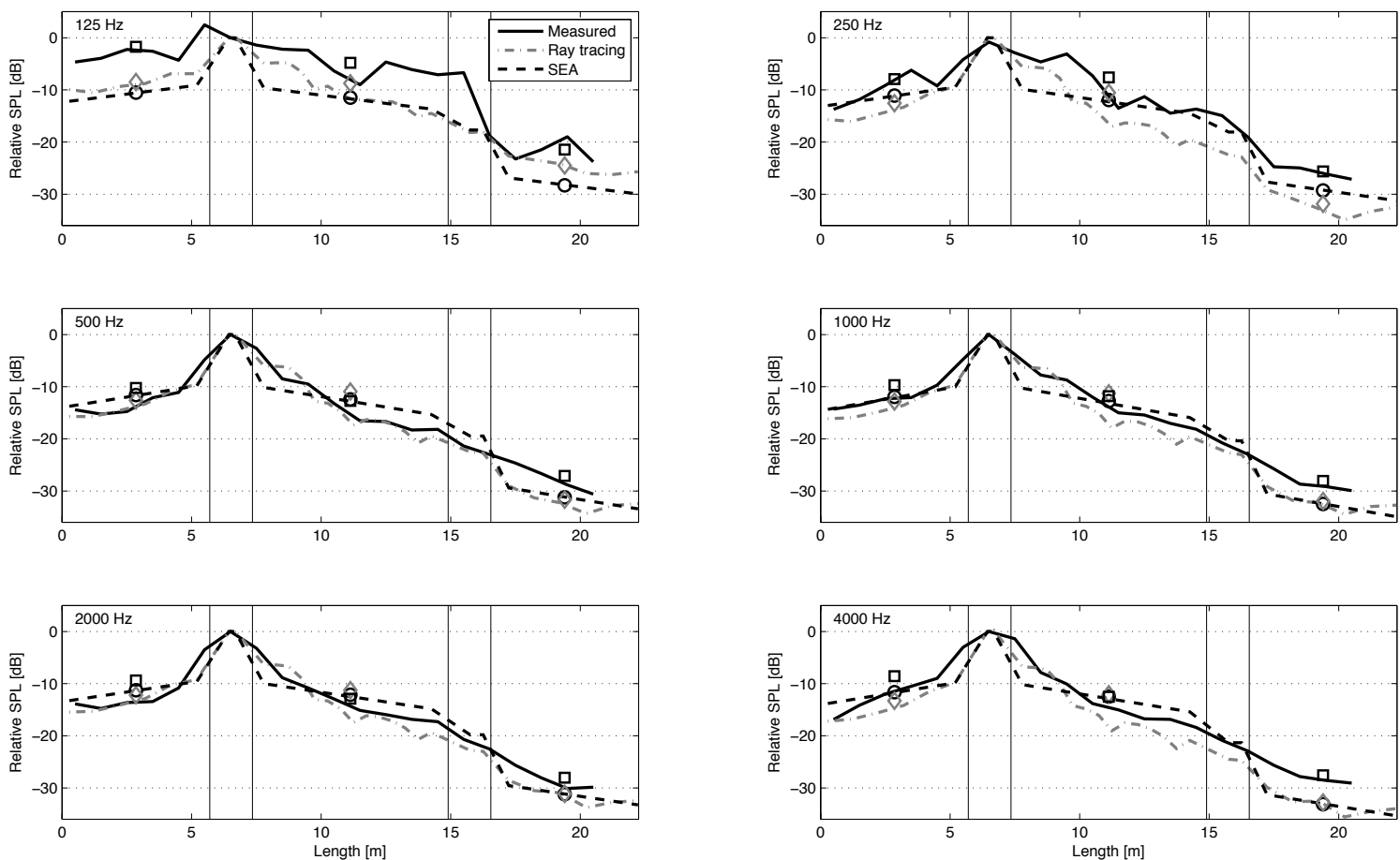

Figure 4. Measured and predicted sound pressure levels relative to Vestibule 1 . The markers show the energy-averaged results for the saloons (squares: measurements; diamonds: ray-tracing; circles: SEA).

\section{Conclusions}

The sound propagation throughout a train car with five compartments (a Swedish Regina train) has been modelled using statistical energy analysis (SEA) and validated by a ray-tracing method and scale model measurements. The five compartments, or air cavities, consist of three saloons with an elongated shape and two vestibules. Connected by openings, the compartments form a series of coupled air cavities, denoted as:

Saloon 1, Vestibule 1, Saloon 2, Vestibule 2 and Saloon 3. The spatial decay of the sound field has been investigated for an acoustic source located in Vestibule 2. A good agreement is shown between the measurements, the ray tracing and the SEA modelling for the octave bands 500-4000 Hz, for Saloons 1 and 2, which are adjacent to the source cavity. Since SEA modelling in essence does not treat a varying sound field within a subsystem, the model has been adjusted using theory from existing literature on the rate of spatial decay in corridors. With this adjustment to the SEA model, the rate of decay was still slightly underestimated compared with the measurements and the ray tracing. It is reasonable to assume that the larger diffusion due to the seating in the saloons calls for this increase in rate of spatial decay. In any case, the average energy was still well predicted by the SEA modelling.

The limiting conditions for the SEA modelling concerning modal density and modal overlap factor are well fulfilled from the $500 \mathrm{~Hz}$ octave band for each of the saloons. However, for the vestibules, the limiting conditions are satisfied only from above $1 \mathrm{kHz}$. Therefore the main part of the SEA results should be treated with care for Vestibule 2, as well as for Saloon 3, which is connected only to Vestibule 2. 
Even though the ray-tracing method gave slightly better agreement with the data from the scale model measurements, the SEA modelling might still be preferable thanks to the simplicity of setting up the model and the insignificant computation time. The underestimated rate of spatial decay of the adjusted SEA modelling used here could hopefully be improved in further work, e.g. by investigating the sound field at multiple points in the cross-section of the train car.

\section{Acknowledgement}

The work behind this paper was partly funded by Bombardier Transportation Sweden AB.

\section{References}

[1] Corakci, A. C. and Tober, S. Modeling of interior sound field in railway vehicles Special focus on sound transmission between vestibules and saloons. MSc Thesis, Applied Acoustics, Chalmers University of Technology, Sweden, 2009.

[2] Kuttruff, H., Room Acoustics (3rd edn), Elsevier Applied Science, London, UK, 1991.

[3] Lyon, R.H. Statistical Energy Analysis of Dynamical Systems, M.I.T. Press, 1975.

[4] Fahy, F. J. A note on the subdivision of a volume of air in a vehicle enclosure into SEA subsystems. Journal of Sound and Vibration, 271, 1170-1174, 2004.

[5] Craik, R. J. M. Sound Transmission Through Buildings Using Statistical Energy Analysis. Gower, England, 1996.

[6] Redmore, T. L. and Flockton, S. J. A design formula for predicting the attenuation of sound along a long corridor. Acoustic Letters, 1, 21-24, 1977.

[7] Hopkins, C. Sound insulation. Elsevier, Oxford, UK, 2007. 